\title{
CD40 ligand deficiency presenting as unresponsive neutropenia
}

\author{
Francis J Andrews, Faye Katz, Alison Jones, Susie Smith, Adam Finn
}

\begin{abstract}
A male child presented with recurrent respiratory infections, otitis media, and oral ulceration and was found to be neutropenic. Investigations showed hypogammaglobulinaemia with normal serum IgM and a novel deletion in the gene for CD40 ligand on his $X$ chromosome. Intravenous gammaglobulin did not lead to resolution of his neutropenia; G-CSF was also necessary. (Arch Dis Child 1996; 74: 458-459)
\end{abstract}

Keywords: hyper IgM syndrome, neutropenia, CD40 ligand deficiency.

The hyper IgM syndrome is characterised by low plasma concentrations of IgG, IgA, and IgE associated with normal or increased levels of IgM. It is usually an $\mathrm{X}$ linked primary immunodeficiency caused by a mutation in the gene for the $T$ cell surface protein $C D 40$ ligand ${ }^{1}$ which is involved in $\mathrm{T}$ cell interaction with $B$ cells and the induction of isotype switching. The condition often presents in the first year of life with recurrent respiratory tract infections and with opportunistic infection, especially with $P$ carinii. In vitro assays of $\mathrm{T}$ cell number and proliferation are normal. Neutropenia is occasionally associated with the syndrome but the reason for this is unclear. Treatment with prophylactic cotrimoxazole and intravenous gammaglobulin prevents infections and is reported to alleviate the neutropenia.

\section{Case report}

A male infant was born by uncomplicated delivery at 42 weeks' gestation and remained well until the age of 6 months. Thereafter he suffered from repeated upper respiratory tract infections, otitis media, mouth ulceration, and oral candida infection. There was no relevant family history. Investigations done at 20 months of age, revealed a haemoglobin $(\mathrm{Hb})$ concentration of $9 \cdot 1 \mathrm{~g} / \mathrm{dl}$ with an MCV of 62.4 $\mathrm{fl}$, an $\mathrm{MCH}$ of $20.4 \mathrm{pg}$, and a neutrophil count of $0.2 \times 10^{9} / 1$. Five subsequent neutrophil counts were less than $0.4 \times 10^{9} / 1$ and a bone marrow aspirate showed maturation arrest at the myelocyte stage with features of an iron deficiency anaemia. A diagnosis of chronic idiopathic neutropenia was made. At 21 months of age, treatment with recombinant human granulocyte colony stimulating factor (GCSF, Roche) was started at a dose of $10 \mu \mathrm{g} / \mathrm{kg}$ once daily, given subcutaneously. After treatment with G-CSF for one week, the neutrophil count had risen to $2.5 \times 10^{9} / 1$ but subsequently continued to fluctuate unpredictably between 0 and $1 \cdot 7$, and the daily dose was progressively increased to $24 \mu \mathrm{g} / \mathrm{kg}$. The patient suffered two further attacks of otitis media. Culture of accompanying aural discharges yielded $S$ pneumoniae and $H$ influenzae. Assays of immunoglobulin isotypes at 24 months of age showed $(\mathrm{g} / \mathrm{l}) \operatorname{IgG} 2 \cdot 1$, IgA $<0 \cdot 2$, $\operatorname{IgM} 1 \cdot 6, \operatorname{IgG}_{1} 1 \cdot 57, \operatorname{IgG}_{2} 0 \cdot 26, \operatorname{IgG}_{3} 0 \cdot 1$, and $\mathrm{IgG}_{4}<0.02 ; \mathrm{IgE}$ was $2 \mathrm{iu} / \mathrm{l}$. With the exception of $\mathrm{IgE}, \mathrm{IgM}, \mathrm{IgG}_{3}$, and $\mathrm{IgG}_{4}$, these values are abnormally low. Circulating numbers of $\mathrm{CD} 3$, CD4, CD8, CD16, and CD19 positive lymphocytes were normal. No anti-neutrophil antibodies were detected and protein electrophoresis of serum showed no abnormalities. Suboptimal responses to previous vaccination against polio, measles, mumps, and rubella were demonstrated, but circulating isohaemagglutinins (anti-A) were detectable. He commenced regular prophylactic cotrimoxazole, but had a further attack of otitis media. $\mathrm{He}$ also developed primary varicella zoster infection; he was treated with intravenous acyclovir and zoster immunoglobulin and made an uneventful recovery.

On the basis of these data a possible diagnosis of hyper-IgM syndrome was suggested and regular treatment with intravenous gammaglobulin (Sandoz) $(0.4 \mathrm{~g} / \mathrm{kg}$ every three weeks) was instituted. Immunofluorescence staining of activated lymphocytes confirmed absence of expression of CD40 ligand (figure, A and B). Genomic DNA from the affected boy was PCRamplified using primers spanning all five exons of the CD40 ligand gene and screened for mutations using single strand conformational polymorphism analysis (SSCP). A band shift was identified in exon 5 (figure, $C$ ), and on subsequent sequencing was found to represent a three-nucleotide deletion (nucleotides 568570 ), which results in loss of an isoleucine residue at position 171 (data not shown).

Despite starting IVGG and continuing treatment with subcutaneous GCSF $24 \mu \mathrm{g} / \mathrm{kg} / \mathrm{d}$ his neutrophil count continued to fluctuate and was often undetectable. His subsequent course was complicated by two central venous line infections, frequent pyrexial illness, and oral ulceration. Eventually an increased dose of GCSF, $33 \mu \mathrm{g} / \mathrm{kg} / \mathrm{d}$, resulted in a persistently normal neutrophil count and an improved clinical course.

\section{Discussion}

The neutropenia that is seen in some cases of hyper IgM syndrome ${ }^{2}$ cannot easily be explained in terms of what is currently known about the function of CD40 ligand but suggests 
(A) Flow cytometric fluorescence histograms of $T$ cells (separated by positive selection using sheep erythrocyte rosetting and density gradient centrifugation) from the patient, following overnight stimulation with phorbol myristate acetate and ionomycin, stained with saturating concentrations of either mouse monoclonal anti-CD40 ligand antibody (5c8) or anti-CD25 antibody (aTac) or no first antibody, followed, after washes, by fluorescein conjugated polyclonal goat anti-mouse serum. (B)

Equivalent histograms from a normal adult control sample processed in parallel. In both, effective $T$ cell activation is demonstrated by detectable CD25 expression, but, in contrast to the control, the patient's cells show no CD40 ligand expression. (C) SSCP analysis of the PCR product of exon 5 of the CD40 ligand gene, digested with BsoF I for patient (track 1) and control (track 2) samples.

The band shift in the single stranded products are indicated by closed arrows above compared to the normal products, indicated by open arrows below.
A

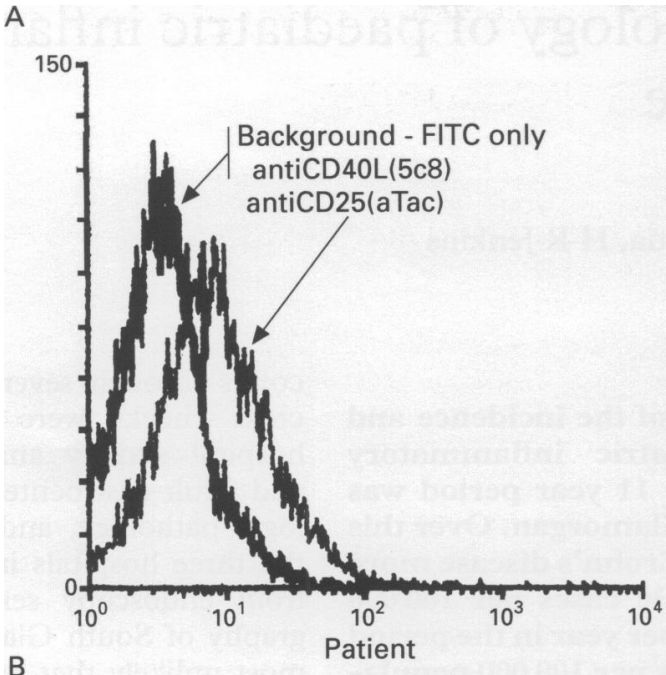

B
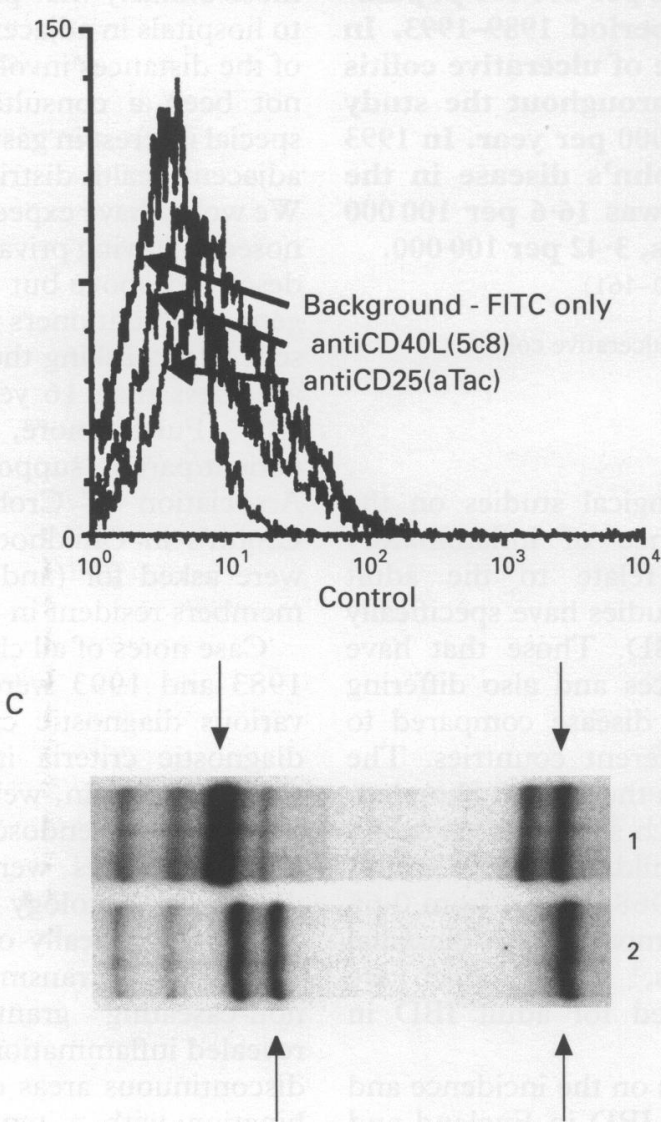

that it plays a role in early myeloid differentiation. ${ }^{3}$ Furthermore, the responsiveness of these neutropenias to treatment varies markedly in reported cases between responding to IVGG alone $^{14}$ or responding to additional GCSF therapy. ${ }^{5}$ In another case, neutropenia was resistant to GCSF and corrected only by bone marrow transplantation. ${ }^{6}$

This case report emphasises the importance of considering the possibility of an underlying defect of specific immunity in a child who presents with recurrent infections and is found to be neutropenic. Heterogeneous mutations in the CD40 ligand gene have been reported to date. If this mutation or closely associated ones are found in other cases of CD40 ligand deficiency with neutropenia, this could shed some light on the mechanism of the association between the two. On the other hand, we have also observed neutropenia in association with a minority of boys with $\mathrm{X}$ linked agammaglobulinaemia which is due to mutations in a different gene coding for a B cell tyrosine kinase, so it may be that neutropenia occurs in occasional hypogammaglobulinaemic patients by a mechanism not directly due to the primary gene defect.

We thank Drs B Paul and L Williams for referring this patient. SS's work is supported by the MRC.

1 Korthauer U, Graf D, Mages HW, et al. Defective expression of T-cell CD40 ligand causes X-linked immunodeficiency with hyper-IgM. Nature 1993; 361: 539-41.

2 Banatvala N, Davies J, Kanariou M, Strobel S, Levinsky $R$ Morgan G. Hypogammaglobulinaemia associated with normal or increased IgM (the hyper IgM syndrome): a case normal or increased IgM (the hyper IgM syndrom

3 Banchereau J, Bazan F, Blanchard D, et al. The CD40 antigen and its ligand. Annu Rev Immunol 1994; 12: 881-922.

4 Rieger CH, Moohr JW, Rothberg RM. Correction of neutropenia associated with dysgammaglobulinemia. Pediatrics 1974; 54: 508-11.

5 Wang WC, Cordoba J, Infante AJ, Conley ME. Successful treatment of neutropenia in the hyper-immunoglobulin $M$ syndrome with oranulocyte colony-stimulating factor. $A m$ f Pediatr Hematol Oncol 1994; 16: 160-3.

6 Fasth A. Bone marrow transplantation for hyper-IgM syndrome. Immunodeficiency 1993; 4: 323. 